\title{
Data-Driven Remaining Useful Life Prediction Based on Domain Adaptation
}

\author{
Bingcheng Wen ${ }^{\text {Corresp., } 1}$, Ming qing Xiao ${ }^{1}$, Xue qi Wang ${ }^{1}$, Xin Zhao $^{1}, \operatorname{Jian}_{\text {feng }} \mathbf{L i}^{1}$, Xin Chen ${ }^{1}$ \\ ${ }^{1}$ ATS Lab, Air Force Engineering University, Xi'an, Shan xi, China \\ Corresponding Author: Bingcheng Wen \\ Email address: 1255292204@qq.com
}

As an important part of prognostics and health management, remaining useful life (RUL) prediction can provide users and managers with system life information and improve the reliability of maintenance systems. Data-driven methods are powerful tools for RUL prediction because of their great modeling abilities. However, most current data-driven studies require large amounts of labeled training data and assume that the training data and test data follow similar distributions. In fact, the collected data are often variable due to different equipment operating conditions, fault modes, and noise distributions. As a result, the assumption that the training data and the test data obey the same distribution may not be valid. In response to the above problems, this paper proposes a data-driven framework with domain adaptability using a bidirectional gated recurrent unit (BGRU). The framework uses a domain-adversarial neural network (DANN) to implement transfer learning (TL) from the source domain to the target domain, which contains only sensor information. To verify the effectiveness of the proposed method, we analyze the IEEE PHM 2012 Challenge datasets and use them for verification. The experimental results show that the generalization ability of the model is effectively improved through the domain adaptation approach. 
1 Data-Driven Remaining Useful Life Prediction Based on

2 Domain Adaptation

3 Bin Cheng Wen ${ }^{1 *}$, Ming Qing Xiao ${ }^{1}$, Xue Qi Wang ${ }^{1}$, Xin Zhao ${ }^{1}$, Jian Feng $\mathrm{Li}^{1}$, Xin Chen ${ }^{1}$

${ }^{1}$ ATS Lab, Air Force Engineering University, Xi'an, 710038, China; 1255292204qq.com

7 Corresponding Author:

8 Bin Cheng Wen ${ }^{1}$

9 'ATS Lab, Air Force Engineering University, Xi' an, 710038, China

10 Email address: 1255292204qq.com

\section{Abstract}

As an important part of prognostics and health management, remaining useful life (RUL) prediction can provide users and managers with system life information and improve the reliability of maintenance systems. Data-driven methods are powerful tools for RUL prediction because of their great modeling abilities. However, most current data-driven studies require large amounts of labeled training data and assume that the training data and test data follow similar distributions. In fact, the collected data are often variable due to different equipment operating conditions, fault modes, and noise distributions. As a result, the assumption that the training data and the test data obey the same distribution may not be valid. In response to the above problems, this paper proposes a data-driven framework with domain adaptability using a bidirectional gated recurrent unit (BGRU). The framework uses a domain-adversarial neural network (DANN) to implement transfer learning (TL) from the source domain to the target domain, which contains only sensor information. To verify the effectiveness of the proposed method, we analyze the IEEE PHM 2012 Challenge datasets and use them for verification. The experimental results show that the generalization ability of the model is effectively improved through the domain adaptation approach.

\section{Introduction}

Prognostics aims to provide reliable remaining useful life (RUL) predictions for critical components and systems via a degradation process. Based on reliable forecast results, managers can determine the best periods for equipment maintenance and formulate corresponding management plans; this is expected to improve reliability during operation and reduce risks and costs. Typically, prognostic methods are classified into model-based methods and data-driven methods [1]. Model-based methods describe the degradation process of engineering systems by establishing mathematical models based on the failure mechanism or the first principle of damage [2]. 
39

40

41

42

43

44

45

46

47

48

49

50

51

52

53

54

55

56

57

58

59

60

61

62

63

64

65

66

67

68

69

70

71

72

73

74

75

76

77

78

environments, so the uncertainty of parameters limits the application of such methods in industrial systems [3].

Different from model-based methods, data-driven methods can construct the mapping relationship between historical data and RUL information but do not need to study the degradation mechanism of the given system. Data-driven methods have become the focus of research due to their powerful modeling capabilities. Among them, machine learning, as a very common data-driven method, has been widely used in the field of RUL prediction. For example, Theodoros $\mathrm{H}$ proposed an E-support vector machine (SVM) method to predict the remaining life of a rolling bearing [4]. To solve the limitations of SVMs, Wang proposed an RUL prediction method based on a relevance vector machine (RVM) [5]. Selina proposed a naive Bayes-based RUL prediction model for lithium-ion batteries [6]. Wu used a random forest (RF) to predict tool wear [7]. However, machine learning methods require manual extraction or signal processing and statistical projection to obtain health factors. On the other hand, feature extraction is separate from parameter training.

Recently, deep neural networks have been widely used in the field of RUL prediction due to their powerful feature extraction capabilities and regression analysis capabilities [8][9][10][11]. Deep learning not only combines feature extraction with the parameter training process but can also automatically learn relevant features instead of manually designing them. This greatly compensates for the shortcomings of machine learning. At the same time, most of the signals collected by the associated sensors are time series. Some deep neural networks that can support sequence data as inputs are also widely used in RUL prediction. For example, recurrent neural networks (RNNs) [12] [13][14], long short-term memory networks (LSTMs) [15], and gated recurrent units (GRUs) [22] are common approaches.

Although data-driven methods based on deep learning have achieved good results on RUL prediction tasks, in such methods, the network needs to be trained with a large number of labeled datasets to obtain a sufficiently accurate model. However, for complex systems, it is often difficult to collect sufficient data with run-to-failure information. Furthermore, the current methods based on deep learning require the training data and the test dataset to follow similar distributions, which means that the dataset needs to come from the same feature space. However, in the actual application process, due to the changing environment in which equipment operates, differences in data distribution are widespread, which leads to a decline in RUL prediction accuracy in actual applications. In other words, the RUL prediction model obtained through the training dataset may not have good generalization ability, and the performance on the test dataset may be poor.

To solve these problems, some domain adaptation methods have been designed gradual application in the field of prognostics and health management (PHM). The purpose of domain adaptation is to use existing knowledge to solve different but related problems. This means that we can use a number of models trained with labeled data to adapt to data with different input characteristics, data distributions, and limited or no labeled data. However, the existing life prediction method based on domain adaptation is difficult to adapt to multivariate sensor data 
79 because it does not consider the temporal dependency problem [28]. As a result, the existing

80 RUL methods based on transfer learning (TL) can hardly adapt to common RUL prediction 81 problems.

82 In this article, we propose the use of bidirectional GRUs (BGRUs) to solve the problem of

83 sequential data processing. We use labeled source domain data and unlabeled target domain data

84 for training. This can be viewed as a process of unsupervised learning based on feature transfer.

85 At the same time, we use a domain-adversarial neural network (DANN) to learn features with

86 domain invariance. To verify the method proposed in this article, we use the IEEE PHM 2012

87 Challenge datasets for verification. The experimental results prove the effectiveness of the 88 method proposed in this article.

89 The main contributions of our work are as follows:

90

91

92

93

(1) We propose a new RUL prediction structure that can better adapt to data distribution shifts under different working environments and fault modes.

(2) The framework not only uses a single sensor but also integrates information from multiple sensors.

(3) Compared with the nonadaptive method and the traditional nondeep adaptive method, our proposed structure obtains better prediction results.

The rest of this article is organized as follows: Section 2 briefly introduces the theoretical background of TL and deep learning. Then, the experimental procedure is introduced in Section 3. In Section 4, the BGRU, DANN, domain-adaptative BGRU and BGRU-DANN structures proposed in this article are introduced. On this basis, RUL prediction for a bearing dataset is studied. The comparative results and conclusions are given in Section 5.

\section{Literature Review}

\section{Deep learning and PHM}

Within the framework of deep learning, RNN is a very representative structure. It can not only process sequence data but also extract features well. Furthermore, RNNs have been used in the field of RUL prediction [16] [17]. However, such networks cannot deal with the weight explosion and gradient disappearance problems caused by recursion. This limits their application in long-term sequence processing. To solve this problem, many RNN variants have begun to appear, for example, LSTM and GRUs. These networks can process series with long-term correlations and extract features from them.

As a variant of the RNN proposed earlier, LSTM has already performed well in RUL prediction. Shi [18] also showed similar results; real-time, high-precision RUL prediction was achieved by training a dual-LSTM network. Chen [19] tried to add the attention mechanism commonly used in the image field to an LSTM network and proposed an attention-based LSTM method, which also achieved good results. Ma [20] proposed integrating deep convolution into the LSTM network. This approach applies a convolution structure to output-to-state and state-to-state information and uses time and time-frequency information simultaneously.

As another type of RNN variant, GRUs have also begun to be applied in RUL prediction. 
119 Compared with LSTM, a GRU has a simpler structure and fewer parameters, but the effect is

120

121

122

123

124

125

126

127

128

129

130

131

132

133

134

135

136

137

138

139

140

141

142

143

144

145

146

147

148

149

150

151

152

153

154

155

156

157

158 comparable to that of LSTM. Deng [21] combined a GRU with a particle filter (PF) and proposed an MC-GRU-based fusion prediction method, which achieved good performance in a prognostic study of ball screws. Lu [22] proposed a GRU network based on an autoencoder. It uses an autoencoder to obtain features and a GRU network to extract sequence information. Compared with standard unidirectional LSTM and GRU, the bidirectional structure can extract better feature information [25]。Huang proposed to combine multi-sensor data with operation data to make RUL prediction based on bidirectional LSTM (BLSTM) [23]. Huang [24] proposed a fusion prediction model based on BLSTM. It not only proves the advantages of LSTM in automatic feature acquisition and fusion, but also demonstrates the excellent performance of BLSTM in RUL prediction. Yu proposed a Bidirectional Recurring Neural Network based on autoencoder for C-MAPSS RUL estimation [25]. She attempted to use BGRU for RUL prediction and validated its effectiveness with Bearing data [26]. There are other deep networks, such as CNN, that are also widely used in the PHM space[27].

\section{Transfer learning}

In most classification or regression tasks, it is assumed that sufficient training data with label information can be obtained. At the same time, it is assumed that the training data and the test data come from the same distribution and feature space. However, in real life, data offset is common. The training data and test data may come from different marginal distributions. As a way to find the similarity between the source domain and the target domain, TL has achieved good results in domain adaptation. The basic TL methods can be divided into the following categories:

(1) Instance-based TL

(2) Feature-based TL

(3) Model-based TL

(4) Relation-based TL

Detailed information about these methods can be found in the literature [29]. In this article, they are divided into two categories according to their development process. One contains nondeep learning methods, and the other is based on deep learning methods.

The most representative nondeep learning approaches are a series of methods based on maximum mean discrepancy (MMD). For example, Pan proposed transfer component analysis (TCA) [30], which is the most representative TL method. Long tried to combine marginal distributions and conditional distributions and proposed joint distribution adaptation (JDA) [31]. Wang believed that marginal distributions and conditional distributions should have different weights. As a result, he proposed balanced distribution adaptation (BDA) [32]. This technique minimizes the distance between the source domain and the target domain through feature mapping so that the data distributions of the two domains can be as similar as possible. There are also some other nondeep learning methods. For example, Tan proposed structural correspondence learning (SCL) [33] based on feature selection. Sun and Gong proposed

Peer] Comput. Sci. reviewing PDF | (CS-2021:04:60537:2:0:NEW 21 Jul 2021) 
159

160

161

162

163

164

165

166

167

168

169

170

171

172

173

174

175

176

177

178

179

180

181

182

183

184

185

186

187

188

189

190

191

192

193

194

195

196

197

198

199

correlation alignment (CORAL) [34] and the geodesic flow kernel (GFK) method [35] based on subspace learning.

With the continuous development of deep learning methods, an increasing number of people are beginning to use deep neural networks for TL. Compared with traditional nondeep TL methods, deep TL has achieved the best results at this stage. The simplest method for conducting deep TL to finetune the deep network, which realizes transfer by finetuning the trained network [36]. At the same time, by adding an adaptive layer to deep learning, deep network adaptation has also begun to appear consistently. For example, Tzeng proposed deep domain confusion (DDC) [37], Ghifary proposed a domain adaptive neural network [38], Long proposed a joint adaptation network (JAN) [39], etc. Recently, as the latest research result in the field of artificial intelligence, generative adversarial networks (GANs) have also begun to be used in transfer learning. Ganin first proposed the DANN [40]. Yu extended a dynamic distribution to an adversarial network and proposed dynamic adversarial adaptation networks (DAANs) [41].

\section{Transfer learning and PHM}

As a way of thinking and a mode of learning, transfer learning has a core problem: finding the similarity between the new problem and the original problem. TL mainly solves the following four contradictions [41]:

(1) The contradiction between big data and less labeling.

(2) The contradiction between big data and weak computing.

(3) The contradiction between a universal model and personalized demand.

(4) The needs of specific applications.

The above four contradictions also exist in PHM. For example, with the development of advanced sensor technology, an increasing amount of data have been collected. However, the amount available data with run-to-failure label information is still small. Second, because the operating state of equipment is affected by many different conditions, the data collected are often not representative due to the differences between various operating conditions and environments. Thus, it is difficult to construct a predictive model with strong universality. Finally, for a PHM system, because of the complexity of the object's use environment, we also need an RUL prediction model with specific applications. However, because there are no data with sufficient label information, it is impossible to use a data-driven approach to build an accurate predictive model. As an effective means, TL can help solve the existing problems of PHM. However, in the field of PHM, TL is mainly used in classification tasks [28]. Shao proposed a convolutional neural network (CNN) based on TL [42], which is used to diagnose bearing faults under different working conditions. Xing proposed a distribution-invariant deep belief network (DIDBN) [43], which can adapt well to new working conditions. Feng pointed out that it is necessary to conduct fault diagnosis research with zero samples [44]. He introduced the idea of zero-shot learning into industrial fields and proposed a zero-sample fault diagnosis method based on the attribute transfer method. RUL prediction studies based on TL are still relatively few in number, as far as the author knows [45][46][47][48]. 


\section{Materials}

\section{Experimental analysis}

202 In this section, we first describe the experimental data and platform in detail. Then, we analyze

203 the data processing and feature extraction methods and introduce the relevant performance

204

205

206

207

208

209

210

211

212

213

214

215

216

217

218

219

220

221

222

223

224

225

226

227

228

229

230

231

232

233

234

235

236

237

238

239

240 metrics. Finally, the effectiveness of our proposed method is verified via a comparison with other methods.

\section{Experimental data description}

The IEEE PHM Challenge 2012 bearing dataset is used to test the effectiveness of the proposed method. This dataset is collected from the PRONOSTIA test platform and contains run-to-failure datasets acquired under different working conditions. PRONOSTIA is composed of three main parts: a rotating part, a degradation generation part and a measurement part. Vibration and temperature signals are gathered during all experiments. The frequency of vibration signal acquisition is $25.6 \mathrm{kHz}$. A sample is recorded every $0.1 \mathrm{~s}$, and the recording interval is $10 \mathrm{~s}$. The frequency of temperature signal acquisition is $10 \mathrm{~Hz} .600$ samples are recorded each minute. To ensure the safety of the laboratory equipment and personnel, the tests are stopped when the amplitude of the vibration signal exceeds $20 \mathrm{~g}$. The basic information of the tested bearing is shown in Table 1. Table 2 gives a detailed description of the datasets. From the table, we can see that the operating conditions of the three datasets are different, and from the literature [48], we can obtain that the failure modes are also different. This is very suitable for experimenting with the method proposed in this article. To verify the effectiveness of the method proposed in this paper, we divide the data into a source domain and target domain according to the different operating conditions. The basic information is shown in Table 3 .

\section{Feature extraction}

The original signal extracted by the sensor cannot reflect the degradation trend of the system well. At the same time, using original data for network training will increase the cost of network training and affect the final output result. It is necessary to extract the degradation information of the system by corresponding methods, which is called feature extraction. From the raw vibration data, we extract 13 basic time-domain features. They are the maximum, minimum, mean, root mean square error (RMSE), mean absolute value, skewness, kurtosis, shape factor, impulse factor, standard deviation, clearance factor, crest factor, and variance. At the same time, through 4-layer wavelet packet decomposition, we extract the energy of 16 frequency bands as time-frequency domain features. In the literature [48], the frequency resolution of the vibration signal was too low. Therefore, we do not extract the frequency domain features but rather use the features of three trigonometric functions. They are the standard deviation of the inverse hyperbolic cosine (SD of the IHC), standard deviation of the inverse hyperbolic sine (SD of the IHS), and standard deviation of the inverse tangent (SD of the IT). For trigonometric features, trigonometric functions transform the input signal into different scales so that the features have better trends [48], and the feature types are shown in Table 4. Through feature extraction, we can obtain 64 features from the feature dataset, which can better 
241 represent the degradation process of the system. Because of space constraints, we only show

242 features along the $\mathrm{X}$-axis of the bearing 1-1 data in Fig. 1.

243

244 Data processing

245 By processing the original data, we extract a set of feature vectors, which are expressed as

$246 X=\left(x_{1}, x_{2}, x_{3}, \ldots \ldots, x_{N}\right)$. To obtain a better experimental result, the experimental data need to be

247 normalized. In this article, the maximum and minimum values are normalized, and the basic

248 calculation formula is as follows:

$$
\ddot{x}_{t}^{j}=\frac{x_{t}^{i, j}-\min \left(x^{j}\right)}{\max \left(x^{j}\right)-\min \left(x^{j}\right)}
$$

250

where $x_{t}^{i, j}$ represents the $i t h$ value of the $j t h$ feature at the $t$ th moment and $x^{j}$ is the $j t h$

251

252

253

254

255

256

257

258

259

260

261

262

263

264

265 MSE:

266

267 R2_score:

268

269

270

271

272 follows.

MAE:

\section{Methods} inputted feature vector.

\section{Sliding time window processing}

After the extracted features are normalized, a sliding time window (TW) is used to generate the time series input $x^{i}=\left\{x_{t}^{i}\right\}_{t=1}^{T_{\omega}}$. The size of the input time window is $T_{\omega}$. The process of its generation is shown in Fig. 2:

\section{Performance metrics}

We use three indicators to evaluate the performance of the proposed method. The mean absolute error (MAE), mean squared error (MSE) and R2_score provide estimations regarding how well the model is performing on the target prediction task. The formulas for their calculation are as

$$
M A E=\frac{1}{L} \sum_{i=1}^{L}\left|y_{i}-\hat{y}_{i}\right|
$$

$$
M S E=\frac{1}{L} \sum_{i=1}^{L}\left(y_{i}-\hat{y}_{i}\right)^{2}
$$

$$
R 2 \text { _score }=1-\frac{\sum_{i=1}^{n}\left(y_{i}-\hat{y}_{i}\right)^{2}}{\sum_{i=1}^{n}\left(y_{i}-\bar{y}^{2}\right)^{2}}
$$

Here, $L$ is the length of the test data, $y_{i}$ is the ith true value, $\hat{y}_{i}$ is the corresponding predicted value, and $\bar{y}$ is the average of the true values. 


\section{Problem definition}

274 We use $T_{S}$ to denote the training task and $T_{T}$ to denote the target task. The training and testing 275 data are represented as the source domain dataset $D_{S}$ and the target domain dataset $D_{T}$,

276 respectively. $D_{S}=\left\{\left(x_{S}^{i}, y_{S}^{i}\right)\right\}_{i=1}^{N_{s}}$, where $x_{S}$ is a series of features belonging to the feature space,

277 its length is $T_{i}$, and its characteristic number is $q_{s} \cdot y_{S}$ represents the RUL label corresponding to

278 the feature sequence $x_{S} . D_{T}=\left\{\left(x_{T}^{i},\right)\right\}_{i=1}^{N_{T}}$, but it only contains characteristic information and no

279 RUL information. We assume that the marginal probability distributions of $D_{S}$ and $D_{T}$ are not

280 the same; that is, $P\left(X_{S}\right) \neq P\left(X_{T}\right)$. Here, we use source and target domain data to learn a

281 prediction function $F$. The goal of the training process is to enable $F$ to estimate the

282 corresponding RUL of the target domain samples during testing. During training, we use the

283 corresponding datasets: $\left\{\left(x_{S}^{i}, y_{S}^{i}\right)\right\}_{i=1}^{N_{S}}$ from the source domain and $\left\{\left(x_{T}^{i}, \quad\right)\right\}_{i=1}^{N_{T}}$ from the target

284 domain. This is an unsupervised TL method. The process of training can be expressed as

$285 y_{S}=F\left(x_{S}, x_{T}\right)$.

286

287 Bidirectional gated recurrent unit

288 A GRU is a variant of the LSTM structure. Compared with LSTM, its structure is simpler, and 289 there are fewer parameters. He combined the forget gate and input gate in LSTM into a single 290 update gate. At the same time, the cell state and hidden state were also merged. A GRU contains 291 two door structures, a reset door and an update door. The reset gate determines whether the new 292 input is combined with the output from the previous moment; that is, the smaller the value of the 293 reset gate is, the less the output information from the previous moment is retained. The update 294 gate determines the degree of influence of the output information from the previous moment on 295 the current moment. The larger the value of the update gate is, the greater the influence of the 296 output from the previous moment on the current output. The GRU-based structure is shown in 297 Fig. 3.

298 In our proposed structure, a BGRU is used to obtain time series features from a TW $T_{W}$. Here, $299 x_{t}$ is the input at time $t$, and $h_{t}$ represents the output of the GRU at time $t . r_{t}$ is the reset gate, 300 and $z_{t}$ is the update gate. These two parts determine how to obtain $h_{t}$ from $h_{t-1}$. The hidden 301 layer of the GRU is defined as follows when running at time $t$ :

302 Forward propagation:

$$
\dot{h}_{t}=f\left(\stackrel{r}{x}_{t}, \grave{h}_{t-1}, \stackrel{\prime}{\theta}_{B G R U}\right)
$$


304

305

306

307

308

309

310

311

312

313

314

315

316

317

318

319

320

321

322

323

324

325

326

327

328

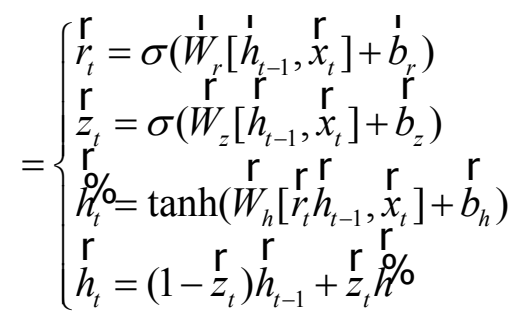

Backward propagation:

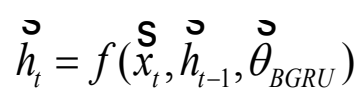

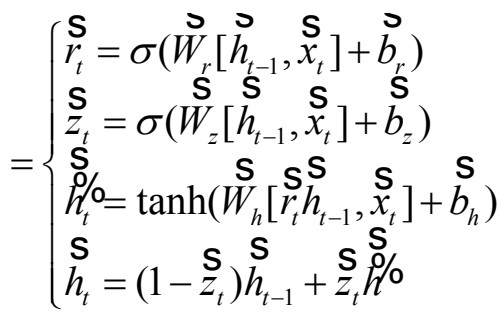

Here, $r_{t}$ controls how much information is passed to $h_{t}$, and its calculation is shown in Eqn. (6) and Eqn. (8). $z_{t}$ determines the extent to which $h_{t-1}$ is passed to the next state, which is calculated as shown in the formula. $\rightarrow, \leftarrow$ represent the processes of forward and backward propagation, respectively. In the forward and backward propagation versions of the formula, $\sigma$ is the sigmoid activation function. $W_{z}$ is the update weight. $W_{r}$ is the reset weight. $b_{r}$ and $b_{z}$ are the deviations. $h_{t}^{/ c}$ indicates the candidate status. $h_{t}$ is the hidden layer output. A portion of the input features of the BGRU can be expressed as $X_{k}=\left(x_{k, 1}, x_{k, 2}, \ldots \ldots, x_{k, t_{W W}}\right)$. The output of the BGRU can be expressed as

$$
\begin{aligned}
H_{k} & =\left[h_{1}, \ldots, h_{t}, \ldots, h_{t_{T W}}\right] \\
& =f\left(X_{k}, \stackrel{\theta}{G}_{G R U}, \theta_{G R U}\right)
\end{aligned}
$$

Here, $f(\bullet)$ represents the hidden layer function of the BGRU, as defined by Eqn. (6) and Eqn. (8). $H_{k}=\left[h_{1}, \ldots, h_{t}, \ldots, h_{t_{T W}}\right]$ is the output characteristic. $\left(\dot{\theta}_{G R U}, \stackrel{\vec{\theta}}{G R U}\right)$ represents the parameters of the forward and backward propagation operations. $h_{t}$ represents the output characteristics, and the formula for the base calculation is as follows:

$$
h_{t}=\dot{h}_{t} \oplus \grave{h}_{t}
$$

\section{Domain adversarial neural networks}

Inspired by the GAN, Yaroslav Ganin first proposed domain-adversarial training for neural networks [40], the process for which is shown in Fig. 4. A DANN combines domain adaptation with feature learning during the training process to better obtain distinctive and domain-invariant features. At the same time, the learned weights can also be directly used in the target field. The network structure of a DANN is mainly composed of three parts: a feature extractor $G_{f}$, a 
329

330

331

332

333

334

335

336

337

338

339

340

341

342

343

344

345

346

347

348

349

350

351

352

353

354

355

356

357

358

359

360

361

category predictor $G_{\mathrm{y}}$ and a domain classifier $G_{d}$. To maximize the domain classification error, $G_{f}$ is used to extract the features with the greatest domain invariance. $G_{\mathrm{y}}$ is used to classify the source domain data. $G_{d}$ is used to distinguish between the characteristic data of the source domain and the target domain. Its training objectives are mainly twofold: the first is to accurately classify the source domain dataset to minimize the category prediction error. The second is to confuse the source domain dataset with the target domain dataset to maximize the domain classification error. The loss function of the DANN can be expressed by the following formula:

$$
L\left(\theta_{f}, \theta_{y}, \theta_{d}\right)=\sum_{\substack{i=1, \ldots N \\ d_{i}=0}} L_{y}\left(G_{y}\left(G_{f}\left(x_{i} ; \theta_{f}\right) ; \theta_{y}\right), y_{i}\right)-\alpha \sum_{i=1, \ldots N} L_{d}\left(G_{d}\left(G_{f}\left(x_{i} ; \theta_{f}\right) ; \theta_{d}\right), y_{i}\right)
$$

Here, $L_{\mathrm{y}}$ is the error of the category predictor, and $L_{d}$ is the error of domain classification. $\theta_{f}$ is the parameter of the feature acquisition layer. The parameter of the category predictor is $\theta_{y} . \theta_{d}$ is the parameter of the domain classifier. During the training process, to find the features with the best domain invariance, on the one hand, it is necessary to find $\theta_{f}$ and $\theta_{y}$ to minimize the category prediction error. On the other hand, it is also necessary to search $\theta_{d}$ to maximize the error of domain classification.

$$
\left(\hat{\theta}_{f}, \hat{\theta}_{y}\right)=\arg \min _{\theta_{f}, \theta_{y}} L\left(\theta_{f}, \theta_{y}, \hat{\theta}_{d}\right)
$$

$$
\left(\hat{\theta}_{d}\right)=\arg \max _{\theta_{d}} L\left(\hat{\theta}_{f}, \hat{\theta}_{y}, \theta_{d}\right)
$$

Judging from the above two optimization formulas, this is a minimax problem. To solve this problem, a gradient reversal layer (GRL) is introduced into the DANN. During the process of forward propagation, the GRL acts as an identity transformation. However, during the back propagation process, the GRL automatically inverts the gradient. The optimization function selected by the DANN is a stochastic gradient descent (SGD) function. The GRL layer is generally placed between the feature extraction layer and the domain classifier layer. The original DANN was the first proposed TL method based on adversarial networks. It is not only a method but also a general framework. Based on these foundations, many people have proposed different architectures [49][50][51].

\section{BGRU-based deep domain adaptation}

To process the time series data, we construct the BGRU-DANN model, the process of which is shown in Fig. 5. Source domain data and target domain data with only domain information are used to train the network. Similar to the DANN network, the BGRU-DANN network can also be divided into three parts. The first part is a feature extraction network. We use a BGRU to map the input data to a hidden state. Then, the output of the BGRU is embedded in the feature space. That is, $f=G_{f}\left(B G R U\left(X_{k}\right), \theta_{f}\right)$. The second part maps the new features to the label data 
362 (source domain) through the fully connected (FC) layer. That is, $\hat{y}=G_{y}\left(f, \theta_{y}\right)$. In the third part,

363 the same feature is mapped to the domain label through the FC layer, i.e., $\hat{d}=G_{d}\left(f, \theta_{d}\right) . G_{f}$

364 consists of a three-layer BGRU and an FC layer. A nonlinear high-dimensional feature

365 representation of the original data is learned through the BGRU and FC layers. $G_{y}$ is composed

366 of FC layers, batch normalization (BN) layers, and a rectified linear unit (ReLU) layer; $G_{y}$

367 provides the regression value of the source domain data. The network form of $G_{y}$ is $\mathrm{FC} 1+\mathrm{BN} 1+$ 368 ReLU1+ Dropout1+ FC2+ BN2+ ReLU2+ FC3.

369 During the adversarial training process, $G_{d}$ is used to distinguish whether the observed feature 370 comes from the source domain or the target domain. $G_{d}$ consists of a gradient reversal layer and 371 three FC layers. Here, $G_{f}$ is trained to extract features so that the difference between the source 372 domain and the target domain is maximized. The labels of the source domain and target domain 373 are set to 1 and 0 , respectively. The loss function of the training process is as follows:

$$
L\left(\theta_{f}, \theta_{y}, \theta_{d}\right)=\frac{1}{n_{s}} \sum_{i=1}^{n_{s}} L_{y}^{i}\left(\theta_{f}, \theta_{y}\right)-\alpha\left(\frac{1}{n_{s}} \sum_{i=1}^{n_{s}} L_{d}^{i}\left(\theta_{f}, \theta_{d}\right)+\frac{1}{n_{t}} \sum_{i=1}^{n_{t}} L_{d}^{i}\left(\theta_{f}, \theta_{d}\right)\right)
$$

375 Here, the loss functions $L_{y}^{i}$ and $L_{d}^{i}$ are defined as:

$$
L_{y}^{i}\left(\theta_{f}, \theta_{y}\right)=\left|\hat{y}_{t}^{i}-y_{t}^{i}\right|^{p}
$$

377

$$
L_{d}^{i}\left(\theta_{d}, \theta_{y}\right)=d^{i} \log \frac{1}{\hat{d}_{t}^{i}}+\left(1-d^{i}\right) \log \frac{1}{1-\hat{d}_{t}^{i}}
$$

378 In the formula, $\hat{y}_{t}^{i}$ is the predicted value of the RUL at time $t$, i.e., $\hat{y}_{t}^{i}=G_{y}\left(f_{t}^{i}, \theta_{y}\right) . d^{i}$ is the 379 field forecast, and $d^{i}=G_{d}\left(f_{t}^{i}, \theta_{d}\right)$. $L_{y}^{i}\left(\theta_{f}, \theta_{y}\right)$ is the regression error. When the value of $p$ is 380 different, different calculation methods can be used. $L_{d}^{i}\left(\theta_{d}, \theta_{y}\right)$ is the binary cross-loss quotient 381 between the domain labels. The optimization process is shown in Eqn. (12) and Eqn. (13). The 382 weight update process is as follows:

$$
\theta_{f} \leftarrow \theta_{f}-\lambda\left(\frac{\partial L_{y}^{i}}{\partial \theta_{f}}-\alpha \frac{\partial L_{d}^{i}}{\partial \theta_{f}}\right)
$$

$$
\theta_{y} \leftarrow \theta_{y}-\lambda \frac{\partial L_{y}^{i}}{\partial \theta_{f}}
$$


386

387

388

389

390

391

392

393

394

395

396

397

398

399

400

401

402

403

404

405

406

407

408

409

410

411

412

413

414

415

416

417

418

419

420

421

422

423

424

425

426

Similar to a DANN, the GRL mechanism is also introduced here to realize the optimization process. SGD is used to update Eqns. (17), (18) and (19).

\section{BGRU-DANN structure}

The structure of BGRU-DANN is shown in Fig. 6. Its basic composition can be divided into two parts. One part uses the training data from the source domain to minimize the loss of source domain regression. The other part uses the sensor data of the source domain and the target domain to maximize the error of domain classification. The BGRU and FC layers are shared by both parts. To facilitate the parameter setting process, we set the learning rates of the two sections to the same value. At the same time, we use dropout and BN layers for feature acquisition, domain classification and source domain regression. In the source domain regression task, the purpose of the training process is to minimize the regression loss function. In the domain classification task, a GRL is placed between the feature extraction and domain classification layers. During the process of back propagation, the GRL inverts the corresponding gradient to realize the optimization process of the model. When the output of the system does not improve significantly, the training process is stopped. For the corresponding FC layer, we use the ReLU activation function.

\section{Results}

\section{Transfer prediction}

To realize the prediction of RUL, we need to establish the BGRU-DANN structure and set the corresponding hyperparameters. For different transfer tasks, the optimal parameters of the model may vary. The model in this paper has no specific optimization process for parameter setting during use, and the parameters used are the same for different transfer tasks. The input size of the BGRU network is set to 64 . The size of each hidden layer is set to 256 . The number of network layers is set to 3. The DANN classifier is set to a 3-layer FC structure, and the domain classifier is a 3-layer FC structure. The network learning rate is set to 0.01 . The number of training iterations is set to 5000. Some of the remaining hyperparameter settings are provided in Table 5 . After setting the relevant parameters, we can predict the RUL. First, we use the BGRU structure to extract the features of the input sequence data. Then, the DANN network is used to implement adversarial training to extract features with domain invariance. The experimental results are shown in Fig. 7 and Fig. 8.

Fig. 7 reflects the predicted results of bearing 2-1, bearing 2-4 and bearing 2-6. The source domain data are bearing 1-3-bearing 1-7, and the target data are bearing 2-1, bearing 2-4, and bearing 2-6. Fig. 8 reflects the prediction results for bearing 3-1, bearing 3-2, and bearing 3-3. The source domain data are bearing 1-3-bearing 1-7, and the target data are bearing 3-1, bearing 3-2, and bearing 3-3. From (A), (C), and (E) in Fig. 7 and (A), (C), and (E) in Fig. 8, we can conclude that the predicted RUL results exhibit a good downward trend performance and are very close to the real RUL values; this effectively illustrates the effectiveness of the proposed data-driven prediction framework based on TL. 
427 Comparison of experimental results

428 To demonstrate the advantages of data-driven prediction methods based on domain adaptation,

429 three methods are used for comparison purposes, namely, a BGRU without transfer learning,

430 TCA-NN, and FC-DANN.

431 We can see in Fig. 7 and Fig. 8 that the RUL prediction results of BGRU-DANN are

432 significantly better than those of the other three methods, and the declining trend can best reflect

433 the real RUL value. However, the other three methods cannot reflect the degradation trend of the

434 RUL effectively.

435 Fig. 9 shows the RUL errors of BGRU-DANN, the BGRU, TCA-NN and FC-DANN. It can be

436 clearly seen from Fig. 9 that the RUL error generated by the BGRU-DANN model is the

437 smallest, especially for bearings 2-4, 3-1 and 3-3. At the same time, bearing 2-1 and bearing 2-6

438 in Fig. 9 clearly reflect that the RUL error generated by BGRU-DANN is smaller than that of the

439 other three methods in most cases. Bearing 3-2 in Fig. 9 may not clearly indicate the superiority

440 of BGRU-DANN due to the large amount of data involved. However, through the comparison of

441 the three evaluation indicators in Table 6, it can still be seen that BGRU-DANN achieves the

442 best effect.

443 Table 6 shows that BGRU-DANN achieves the best results in terms of the three evaluations, the

444 MAE, MSE, and R2_score, which further proves the effectiveness of the method proposed in this

445 paper. Regarding the MSE, the calculated results of the proposed method for bearing 2-1,

446 bearing $2-4$, bearing $2-6$, bearing $3-1$, bearing $3-2$ and bearing $3-3$ are $0.0283,0.0193,0.0217$,

$4470.0298,0.0503$, and 0.0472 , respectively, which are far less than the calculated error results of

448 the other three methods. For the MAE, the calculated results of the proposed method for bearing

449 2-1, bearing 2-4, bearing 2-6, bearing 3-1, bearing 3-2 and bearing 3-3 are 0.1157, 0.0928,

$4500.0875,0.1215,0.1569$, and 0.1238 , respectively, which are still better than the calculated error

451 results of the other three models. In terms of the R2-score calculation results, the calculated

452 results of the proposed method for bearing 2-1, bearing 2-4, bearing 2-6, bearing 3-1, bearing 3-2

453 and bearing $3-3$ are $0.6576,0.7664,0.7367,0.6379,0.3935$, and 0.4252 , respectively; this

454 indicates that the model has certain explanatory ability regarding the relationship between the

455 independent variable and the dependent variable in the regression analysis and is superior to the

456 three compared methods.

457

458 Conclusions

459 In this article, a domain-adaptative prediction method based on deep learning with a BGRU and

460 a DANN is proposed. The validity of the proposed method is demonstrated by an experiment on

461 the 2012 IEEE PHM dataset. The objective of this study is to propose a domain-adaptive RUL

462 prediction method. When the input bearing is transferred from the source domain with label

463 information to a target domain with only sensor information, a more accurate estimate of the

464 RUL can be obtained. From the results of the experiment, we can draw the following conclusions: 
465 (1) Compared with the BGRU without TL, the proposed method has a better effect in terms of 466 RUL prediction. This indicates that the model obtained by adversarial training has better 467 generalization ability and can adapt to data with different distributions.

468 (2) The comparison with TCA-NN proves that the deep, domain-adaptive BGRU-DANN method 469 has better performance. This indicates that the transfer method based on deep learning has a 470 stronger feature extraction ability than the traditional nondeep transfer method, and it can extract 471 better features with domain invariance.

472 (3) Using FC layers for feature extraction, this paper constructs an FC-DANN network. A 473 comparison of the results fully shows that the BGRU has a better effect in terms of feature

474 extraction. Compared with the features extracted by the FC method, the features extracted by the 475 BGRU for sequence data processing are more representative.

476 (4) By means of domain adaptation, the generalization ability of the data-driven RUL prediction 477 model can be effectively improved, and it can adapt to RUL prediction tasks under different 478 working conditions to a certain extent.

479 In future work, we will take a closer look at the problem of time series transfer. Remaining life 480 prediction problems with respect to bearings, aero engines, etc. can actually be regarded as time 481 series transfer problems. However, research on time series transfer is still in its infancy. There 482 are merely a few studies on such issues. Only Yu proposed two different time series transfer 483 methods in references [52] and [53], one based on an extreme learning machine and the other 484 based on a CNN. However, most of the data monitored by sensors are time series data, and this is 485 a very common data type in RUL forecasting research. Therefore, the authors intend to conduct 486 related research in the future, hoping to obtain a better model and research results with more 487 practical application value.

488

489

490

\section{Additional Information And Declarations}

491 Bin Cheng Wen and Ming Qing Xiao, methodology; Bin Cheng Wen, software; Xin Zhao, 492 validation; Bin Cheng Wen, Jian Feng Li and Xin Chen, formal analysis; Jian Feng Li,

493 investigation; Xin Chen., resources; Xin Zhao, data curation; Bin Cheng Wen, preparation for 494 writing the original draft; Jian Feng Li, writing review and editing; Xin Chen, visualization; Xue 495 Qi Wang, supervision.

496

497 Funding

498 This research received no external funding.

499

500

501

Institutional review board statement

502

503

The study did not involve humans or animals.

\section{Data availability statement}

504 The data and code are available at https://github.com/WEN-AFK/BGRU-DANN. 
506

507

508

509

510

511

512

513

514

515

516

517

518

519

520

521

522

523

524

525

526

527

528

529

530

531

532

533

534

535

536

537

538

539

540

541

542

543

544

545

546

547

548

549

550

\section{Conflicts of interest}

The authors declare no conflicts of interest.

\section{References}

[1] A. Heng, S. Zhang, A. C. C. Tan, and J. Mathew, "Rotating machinery prognostics: State of the art, challenges and opportunities," Mechanical Systems and Signal Processing, vol. 23, no. 3, pp. 724-739, 2009.

[2] A. Cubillo, S. Perinpanayagam, and M. Esperon-Miguez, "A review of physics-based models in prognostics: Application to gears and bearings of rotating machinery," Advances in Mechanical Engineering, vol. 8, no. 8, 2016.

[3] M. Pecht, and R. Jaai, "A prognostics and health management roadmap for information and electronics-rich systems," Microelectronics Reliability, vol. 50, no. 3, pp. 317-323, 2010.

[4] T. H. Loutas, D. Roulias, and G. Georgoulas, "Remaining Useful Life Estimation in Rolling bearings Utilizing Data-Driven Probabilistic E-Support Vectors Regression," IEEE Transactions on Reliability, vol. 62, no. 4, pp. 821-832, 2013.

[5] P. Wang, B. D. Youn, and C. Hu, "A generic probabilistic framework for structural health prognostics and uncertainty management," Mechanical Systems and Signal Processing, vol. 28, pp. 622-637, 2012.

[6] S. S. Y. Ng, Y. Xing, and K. L. Tsui, "A naive Bayes model for robust remaining useful life prediction of lithium-ion battery," Applied Energy, vol. 118, pp. 114-123, 2014.

[7] D. Wu, C. Jennings, J. Terpenny, R. X. Gao, and S. Kumara, "A Comparative Study on Machine Learning Algorithms for Smart Manufacturing: Tool Wear Prediction Using Random Forests," Journal of Manufacturing Science and Engineering, vol. 139, no. 7, 2017.

[8] J. Zhu, N. Chen, and W. Peng, "Estimation of bearing Remaining Useful Life Based on Multiscale Convolutional Neural Network," IEEE Transactions on Industrial Electronics, vol. 66, no. 4, pp. 3208-3216, 2019.

[9] X. Li, Q. Ding, and J.-Q. Sun, "Remaining useful life estimation in prognostics using deep convolution neural networks," Reliability Engineering \& System Safety, vol. 172, pp. 1-11, 2018.

[10] J. Deutsch, and D. He, "Using Deep Learning-Based Approach to Predict Remaining Useful Life of Rotating Components," IEEE Transactions on Systems, Man, and Cybernetics: Systems, vol. 48, no. 1, pp. 11-20, 2018.

[11] Y. Wu, M. Yuan, S. Dong, L. Lin, and Y. Liu, "Remaining useful life estimation of engineered systems using vanilla LSTM neural networks," Neurocomputing, vol. 275, pp. 167-179, 2018.

[12]A. Malhi, R. Yan, and R. X. Gao, "Prognosis of Defect Propagation Based on Recurrent Neural Networks," IEEE Transactions on Instrumentation and Measurement, vol. 60, no. 3, pp. 703-711, 2011.

[13] F. O. Heimes, and Ieee, Recurrent Neural Networks for Remaining Useful Life Estimation, 2008.

[14] N. Gugulothu, T. Vishnu, P. Malhotra, L. Vig, P. Agarwal, and G. M. J. A. Shroff, "Predicting Remaining Useful Life Using Time Series Embeddings Based on Recurrent Neural Networks," vol. abs/1709.01073, 2017.

[15] Y. Zhang, R. Xiong, H. He, and M. G. Pecht, "Long Short-Term Memory Recurrent Neural Network for Remaining Useful Life Prediction of Lithium-Ion Batteries," IEEE Transactions

Peer] Comput. Sci. reviewing PDF | (CS-2021:04:60537:2:0:NEW 21 Jul 2021) 
551

552

553

554

555

556

557

558

559

560

561

562

563

564

565

566

567

568

569

570

571

572

573

574

575

576

577

578

579

580

581

582

583

584

585

586

587

588

589

590

591

592

593

594

595

596

on Vehicular Technology, vol. 67, no. 7, pp. 5695-5705, 2018.

[16] W. Yu, I. I. Y. Kim, and C. Mechefske, "An improved similarity-based prognostic algorithm for RUL estimation using an RNN autoencoder scheme," Reliability Engineering \& System Safety, vol. 199, 2020.

[17] L. Guo, N. Li, F. Jia, Y. Lei, and J. Lin, "A recurrent neural network based health indicator for remaining useful life prediction of bearings," Neurocomputing, vol. 240, pp. 98-109, 2017.

[18]Z. Shi, and A. Chehade, "A dual-LSTM framework combining change point detection and remaining useful life prediction," Reliability Engineering \& System Safety, vol. 205, 2021.

[19] Z. Chen, M. Wu, R. Zhao, F. Guretno, R. Yan, and X. Li, "Machine Remaining Useful Life Prediction via an Attention-Based Deep Learning Approach," IEEE Transactions on Industrial Electronics, vol. 68, no. 3, pp. 2521-2531, 2021.

[20] M. Ma, and Z. Mao, "Deep-Convolution-Based LSTM Network for Remaining Useful Life Prediction," IEEE Transactions on Industrial Informatics, vol. 17, no. 3, pp. 1658-1667, 2021.

[21] Y. Deng, D. Shichang, J. Shiyao, Z. Chen, and X. Zhiyuan, "Prognostic study of ball screws by ensemble data-driven particle filters," Journal of Manufacturing Systems, vol. 56, pp. 359-372, 2020.

[22] Y.-W. Lu, C.-Y. Hsu, and K.-C. Huang, "An Autoencoder Gated Recurrent Unit for Remaining Useful Life Prediction," Processes, vol. 8, no. 9, 2020.

[23]C.-G. Huang, H.-Z. Huang, and Y.-F. Li, "A Bidirectional LSTM Prognostics Method Under Multiple Operational Conditions," IEEE Transactions on Industrial Electronics, vol. 66, no. 11, pp. 8792-8802, 2019.

[24] C.G. Huang, X. Yin, H.Z. Huang, and Y.-F. Li, “An Enhanced Deep Learning-Based Fusion Prognostic Method for RUL Prediction," IEEE Transactions on Reliability, vol. 69, no. 3, pp. 1097-1109, 2020.

[25]W. Yu, I. I. Y. Kim, and C. Mechefske, "Remaining useful life estimation using a bidirectional recurrent neural network based autoencoder scheme," Mechanical Systems and Signal Processing, vol. 129, pp. 764-780, 2019.

[26]D. She, and M. Jia, "A BiGRU method for remaining useful life prediction of machinery," Measurement, vol. 167, 2021.

[27]X. Wang, T. Wang, A. Ming, W. Zhang, A. Li, and F. Chu, "Spatiotemporal non-negative projected convolutional network with bidirectional NMF and 3DCNN for remaining useful life estimation of bearings," Neurocomputing, vol. 450, pp. 294-310, 2021.

[28] P. R. d. O. da Costa, A. Akçay, Y. Zhang, and U. Kaymak, "Remaining useful lifetime prediction via deep domain adaptation," Reliability Engineering \& System Safety, vol. 195, 2020.

[29] S. J. Pan, and Q. Yang, "A Survey on Transfer Learning," Ieee Transactions on Knowledge and Data Engineering, vol. 22, no. 10, pp. 1345-1359, Oct, 2010.

[30] S. J. Pan, I. W. Tsang, J. T. Kwok, and Q. Yang, "Domain adaptation via transfer component analysis," IEEE Trans Neural Netw, vol. 22, no. 2, pp. 199-210, Feb, 2011.

[31] M. Long, J. Wang, G. Ding, J. Sun, and P. S. Yu, "Transfer feature learning with joint distribution adaptation." In Proceedings of the Proceedings of the IEEE international conference on computer vision, 2013; pp. 2200-2207.

[32] J. Wang, Y. Chen, S. Hao, W. Feng, and Z. Shen, "Balanced Distribution Adaptation for Transfer Learning," in 2017 IEEE International Conference on Data Mining (ICDM), 2017, 
597

598

599

600

601

602

603

604

605

606

607

608

609

610

611

612

613

614

615

616

617

618

619

620

621

622

623

624

625

626

627

628

629

630

631

632

633

634

635

636

637

638

639

640

641

642

pp. 1129-1134.

[33] S. Tan, and Y. Wang, "Weighted SCL model for adaptation of sentiment classification," Expert Systems with Applications, vol. 38, no. 8, pp. 10524-10531, 2011.

[34] B. Sun, and K. Saenko, "Deep coral: Correlation alignment for deep domain adaptation." In Proceedings of the European conference on computer vision, 2016; pp. 443-450.

[35] B. Gong, Y. Shi, F. Sha, and K. Grauman, "Geodesic flow kernel for unsupervised domain adaptation." In Proceedings of the 2012 IEEE conference on computer vision and pattern recognition, 2012; pp. 2066-2073.

[36] A. S. Razavian, H. Azizpour, J. Sullivan, S. Carlsson, and Ieee, "CNN Features off-theshelf: an Astounding Baseline for Recognition," 2014 Ieee Conference on Computer Vision and Pattern Recognition Workshops, IEEE Computer Society Conference on Computer Vision and Pattern Recognition Workshops, pp. 512-519, 2014.

[37] E. Tzeng, J. Hoffman, N. Zhang, K. Saenko, and T. J. a. p. a. Darrell, "Deep domain confusion: Maximizing for domain invariance," 2014.

[38] M. Long, Y. Cao, J. Wang, and M. Jordan, "Learning transferable features with deep adaptation networks." In Proceedings of the International conference on machine learning, 2015; pp. 97-105.

[39] M. Long, H. Zhu, J. Wang, and M. I. Jordan, "Deep transfer learning with joint adaptation networks." In Proceedings of the International conference on machine learning, 2017; pp. 2208-2217.

[40] Y. Ganin, E. Ustinova, H. Ajakan, P. Germain, H. Larochelle, F. Laviolette, M. Marchand, and V. Lempitsky, "Domain-Adversarial Training of Neural Networks," Domain Adaptation in Computer Vision Applications, Advances in Computer Vision and Pattern Recognition, pp. 189-209, 2017.

[41] C. Yu, J. Wang, Y. Chen, and M. Huang, "Transfer learning with dynamic adversarial adaptation network." In Proceedings of the 2019 IEEE International Conference on Data Mining (ICDM), 2019; pp. 778-786.

[42] H. Shao, M. Xia, G. Han, Y. Zhang, and J. J. I. T. o. I. I. Wan, "Intelligent fault diagnosis of rotor-bearing system under varying working conditions with modified transfer $\mathrm{CNN}$ and thermal images," 2020.

[43] S. Xing, Y. Lei, S. Wang, and F. Jia, "Distribution-Invariant Deep Belief Network for Intelligent Fault Diagnosis of Machines Under New Working Conditions," IEEE Transactions on Industrial Electronics, vol. 68, no. 3, pp. 2617-2625, 2021.

[44] L. Feng, and C. Zhao, "Fault Description Based Attribute Transfer for Zero-Sample Industrial Fault Diagnosis," IEEE Transactions on Industrial Informatics, vol. 17, no. 3, pp. 1852-1862, 2021.

[45] Y. Fan, S. Nowaczyk, and T. Rögnvaldsson, "Transfer learning for remaining useful life prediction based on consensus self-organizing models," Reliability Engineering \& System Safety, vol. 203, 2020.

[46] W. Mao, J. He, and M. J. Zuo, "Predicting Remaining Useful Life of Rolling bearings Based on Deep Feature Representation and Transfer Learning," IEEE Transactions on Instrumentation and Measurement, vol. 69, no. 4, pp. 1594-1608, 2020.

[47] C. Sun, M. Ma, Z. Zhao, S. Tian, R. Yan, and X. Chen, "Deep Transfer Learning Based on Sparse Autoencoder for Remaining Useful Life Prediction of Tool in Manufacturing," IEEE Transactions on Industrial Informatics, vol. 15, no. 4, pp. 2416-2425, 2019.

[48] J. Zhu, N. Chen, and C. Shen, "A new data-driven transferable remaining useful life 
643 prediction approach for bearing under different working conditions," Mechanical Systems 644 and Signal Processing, vol. 139, 2020.

645 [49] E. Tzeng, J. Hoffman, K. Saenko, and T. Darrell, "Adversarial Discriminative Domain 646 Adaptation," in 2017 IEEE Conference on Computer Vision and Pattern Recognition 647 (CVPR), 2017, pp. 2962-2971.

648 [50] J. Shen, Y. Qu, W. Zhang, and Y. Yu, "Wasserstein distance guided representation learning 649 for domain adaptation," In Proceedings of the Proceedings of the AAAI Conference on $650 \quad$ Artificial Intelligence, 2018.

651 [51]Z. Meng, Z. Chen, V. Mazalov, J. Li, and Y. Gong, "Unsupervised adaptation with domain 652 separation networks for robust speech recognition." In Proceedings of the 2017 IEEE Automatic Speech Recognition and Understanding Workshop (ASRU), 2017; pp. 214-221.

[52] R. Ye, and Q. Dai, "A novel transfer learning framework for time series forecasting," Knowledge-Based Systems, vol. 156, pp. 74-99, 2018.

[53] R. Ye, and Q. Dai, "Implementing transfer learning across different datasets for time series forecasting," Pattern Recognition, vol. 109, 2021. 


\section{Table $\mathbf{1}$ (on next page)}

Table_1-Test_data_information 
Table 1. Test data information

\begin{tabular}{cccc}
\hline Pitch diameter & Diameter of the roller & Number of rollers & Contact angle \\
\hline $25.6 \mathrm{~mm}$ & $3.5 \mathrm{~mm}$ & 13 & 00 \\
\hline
\end{tabular}

2 


\section{Table 2 (on next page)}

Table_2-Descriptions_of_the_experimental_datasets 
Table 2. Descriptions of the experimental datasets

\begin{tabular}{cccc}
\hline & Dataset 1 & Dataset 2 & Dataset 3 \\
\hline Load (N) & 4000 & 4200 & 5000 \\
\hline Speed (rpm) & 1800 & 1650 & 1500 \\
\hline & bearing 1-1 & bearing 2-1 & \\
& bearing 1-2 & bearing 2-2 & \\
& bearing 1-3 & bearing 2-3 & bearing 3-1 \\
Dataset & bearing 1-4 & bearing 2-4 & bearing 3-2 \\
& bearing 1-5 & bearing 2-5 & bearing 3-3 \\
& bearing 1-6 & bearing 2-6 & \\
& bearing 1-7 & bearing 2-7 & \\
\hline
\end{tabular}

2 


\section{Table 3 (on next page)}

Table_3-Transfer_prognostics_task 
Table 3. Transfer prognostics task

\begin{tabular}{lll}
\hline Transfer prognostics & \multicolumn{1}{c}{ Source } & \multicolumn{1}{c}{ Target } \\
\hline \multirow{2}{*}{ Dataset 1-Dataset 2 } & Labeled: bearing 1-3-bearing 1-7 & Unlabeled: bearing 2-1 \\
\cline { 3 - 3 } & & Unlabeled: bearing 2-4 \\
Dataset 1-Dataset 3 & Labeled: bearing 1-3-bearing 1-7 & Unlabeled: bearing 2-6 \\
\cline { 3 - 3 } & & Unlabeled: bearing 3-1 bearing 3-2 \\
\cline { 3 - 3 } & & Unlabeled: bearing 3-3 \\
\hline
\end{tabular}

2 


\section{Table 4 (on next page)}

Table_4-Feature_Set 
Table 4. Feature set

\begin{tabular}{cll}
\hline \multicolumn{1}{c}{ Type } & \multicolumn{2}{c}{ Feature } \\
\hline & F1: Maximum & F8: Shape Factor \\
& F2: Minimum & F9: Impulse Factor \\
& F3: Mean & F10: Standard Deviation \\
Time-domain features & F4: RMSE & F11: Clearance Factor \\
& F5: Mean Absolute Value & F12: Crest Factor \\
& F6: Skewness & F13: Variance \\
& F7: Kurtosis & \\
\hline Time-frequency domain & F14-F29: Energies of sixteen bands \\
features & F30: SD of the IHC & \\
\hline & F31: SD of the IHS & \\
\hline & F32: SD of the IT & \\
\hline
\end{tabular}

2 


\section{Table 5 (on next page)}

Table_5-Hyperparameter_settings 
Table 5. Hyperparameter settings

\begin{tabular}{|c|c|c|c|c|c|}
\hline $\begin{array}{c}\text { BGRU } \\
\text { Layers, (Units), [Dropout] }\end{array}$ & F (Units) & $\begin{array}{c}\text { Source Regression } \\
\text { Layers, (Units), } \\
\text { [Dropout] }\end{array}$ & $\begin{array}{c}\text { Domain Classification } \\
\text { Layers, (Units), } \\
\text { [Dropout] }\end{array}$ & $\boldsymbol{\alpha}$ & $\boldsymbol{\lambda}$ \\
\hline $3,(64,256),[0.5]$ & $(256)$ & $3,(256,128,32),[0.5]$ & $3,(256,128,32),[0.5]$ & 0.5 & 0.01 \\
\hline
\end{tabular}

2 


\section{Table 6(on next page)}

Table_6-Performance_metrics_for_the_datasets 
Table 6. Performance metrics for the datasets

\begin{tabular}{|c|c|c|c|c|c|}
\hline Dataset & Performance metric & The proposed method & BGRU & TCA-NN & FC-DANN \\
\hline bearing 2-1 & \multirow{6}{*}{ MSE } & 0.0283 & 1.4652 & 0.5205 & 0.2865 \\
\hline bearing 2-4 & & 0.0193 & 1.5442 & 1.8181 & 1.0214 \\
\hline bearing 2-6 & & 0.0217 & 1.0589 & 1.4436 & 0.9164 \\
\hline bearing 3-1 & & 0.0298 & 2.1957 & 4.4414 & 1.3557 \\
\hline bearing 3-2 & & 0.0503 & 0.0883 & 0.0796 & 0.0606 \\
\hline bearing 3-3 & & 0.0472 & 8.9927 & 8.8676 & 2.9564 \\
\hline bearing 2-1 & \multirow{6}{*}{ MAE } & 0.1157 & 0.9440 & 0.6316 & 0.4218 \\
\hline bearing 2-4 & & 0.0928 & 1.0940 & 1.2917 & 0.9429 \\
\hline bearing $2-6$ & & 0.0875 & 0.8491 & 1.0226 & 0.8063 \\
\hline bearing 3-1 & & 0.1215 & 1.3532 & 1.9420 & 1.0884 \\
\hline bearing 3-2 & & 0.1569 & 0.2070 & 0.2377 & 0.2035 \\
\hline bearing 3-3 & & 0.1238 & 2.5813 & 2.6589 & 1.5394 \\
\hline bearing 2-1 & \multirow{6}{*}{ R2_score } & 0.6576 & -16.6992 & -5.2325 & -2.4311 \\
\hline bearing $2-4$ & & 0.7664 & -17.6799 & -20.7599 & -11.2249 \\
\hline bearing $2-6$ & & 0.7367 & -11.8176 & -16.2749 & -9.9658 \\
\hline bearing 3-1 & & 0.6379 & -25.6589 & -52.0910 & -15.2054 \\
\hline bearing 3-2 & & 0.3935 & -0.06367 & 0.0451 & 0.2733 \\
\hline bearing 3-3 & & 0.4252 & -108.42439 & -104.9228 & -34.3136 \\
\hline
\end{tabular}


Figure 1

Figure1. Features for Bearing 1-1 

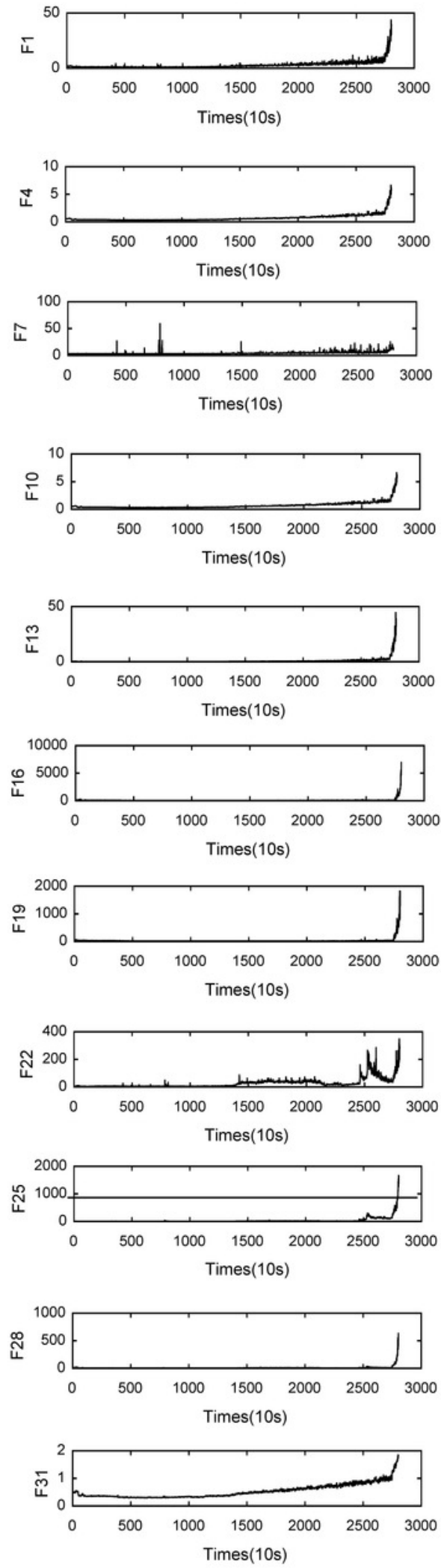
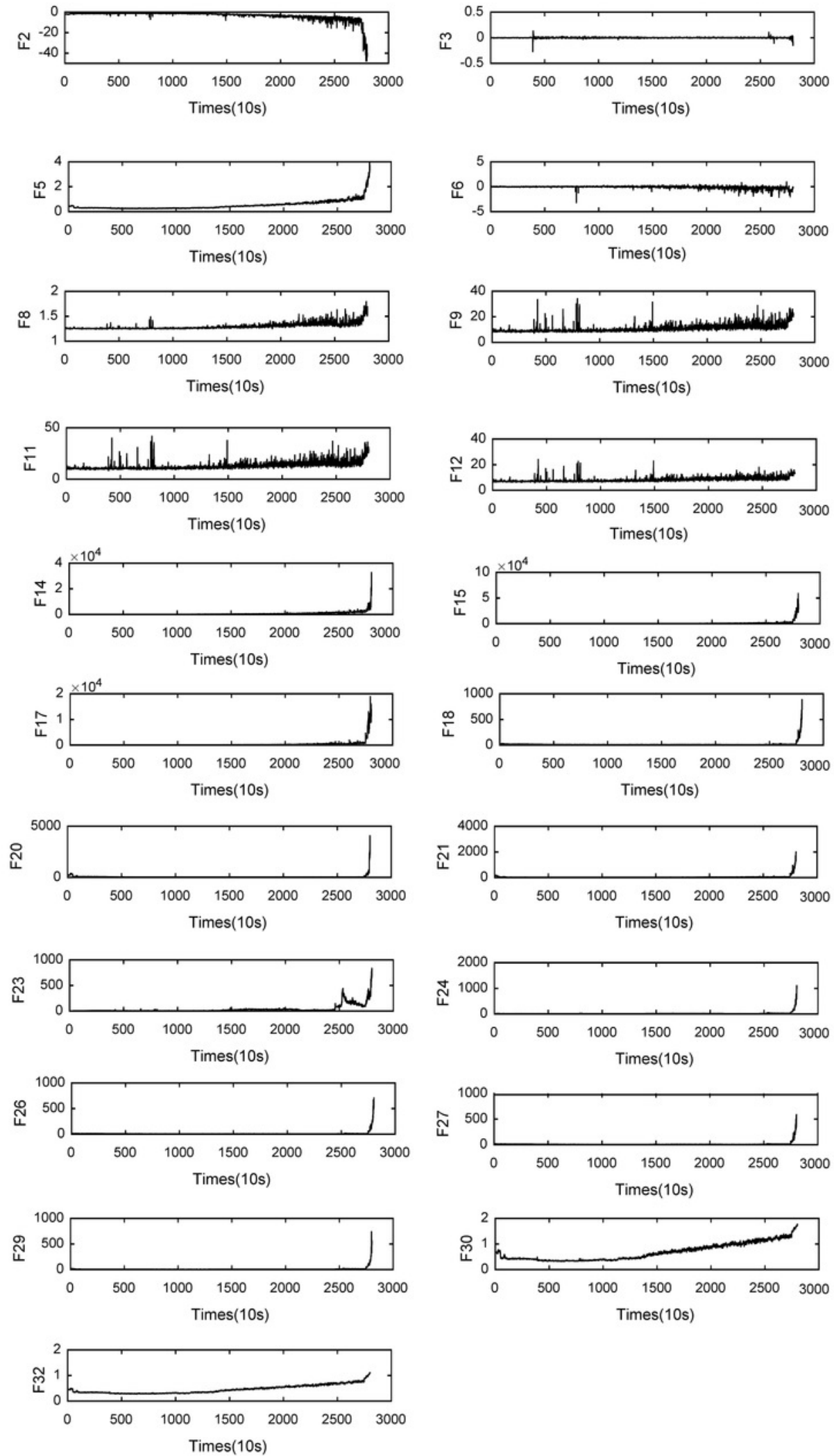
Figure 2

Figure 2. Sliding TW processing technique

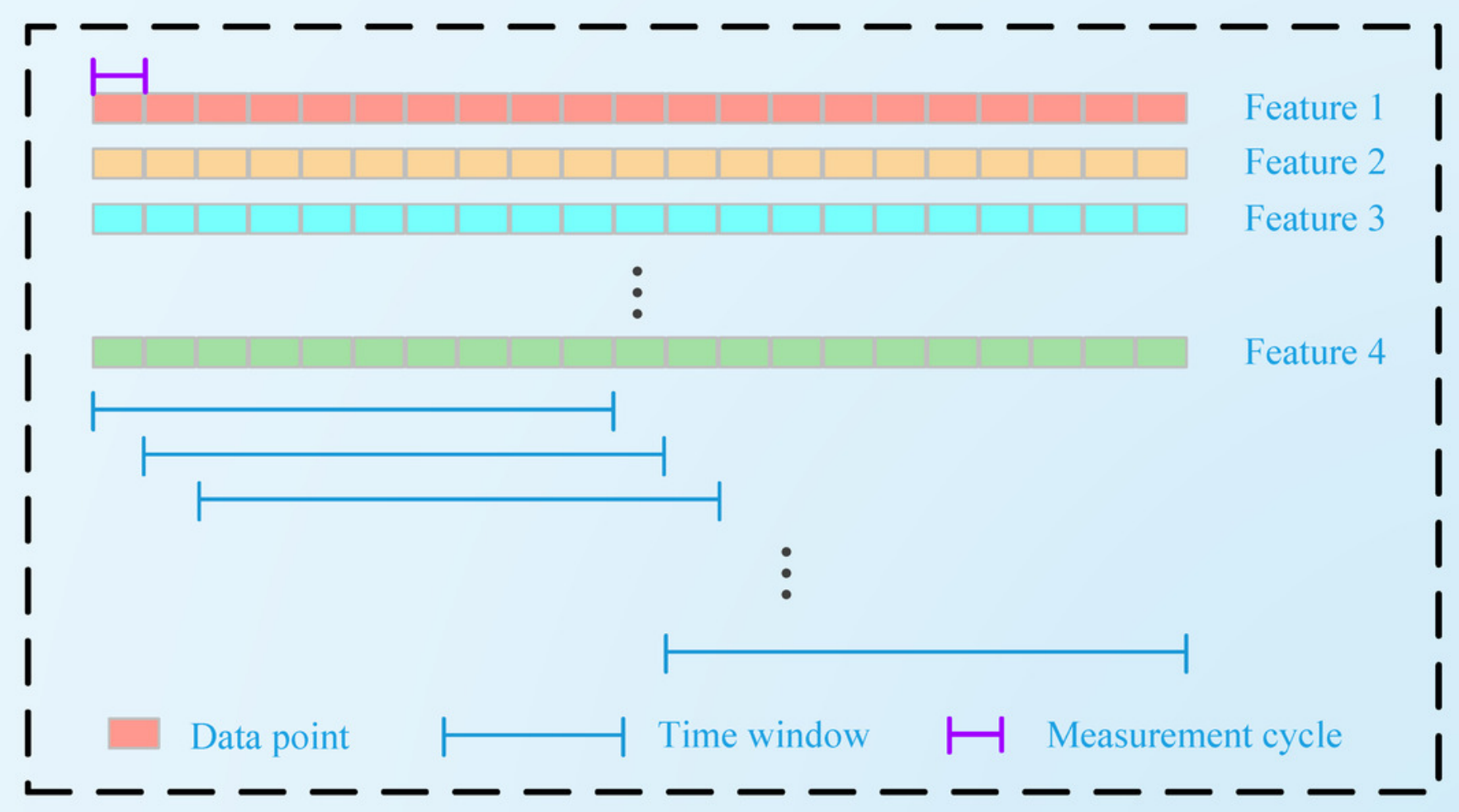


Figure 3

Figure 3. GRU memory cell

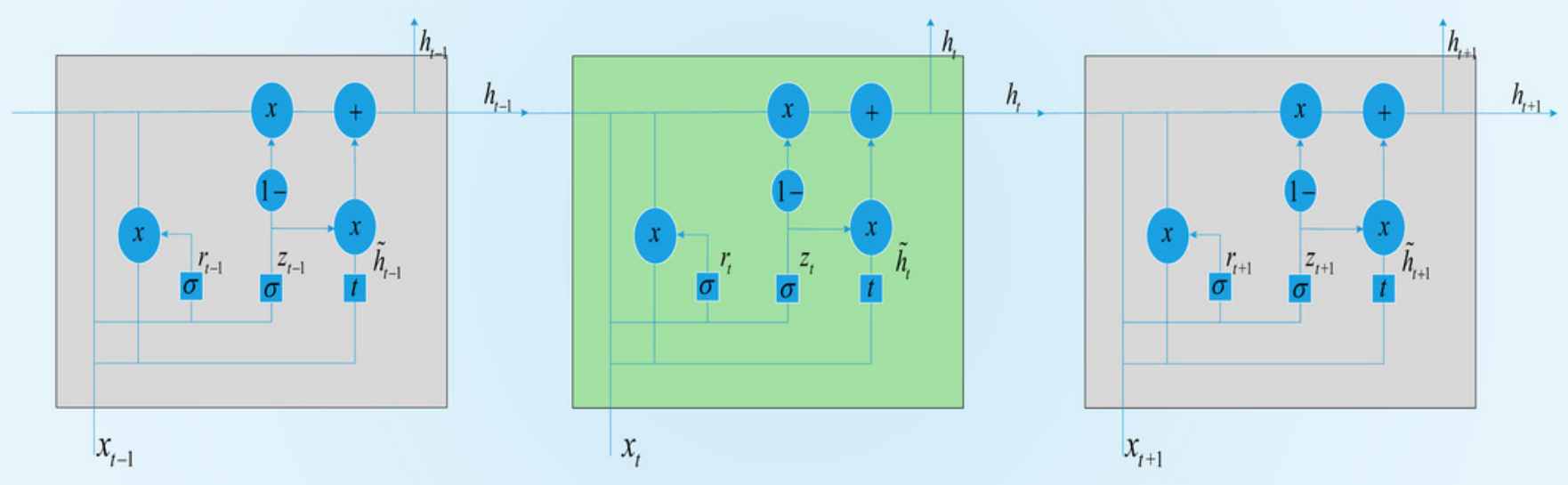


Figure 4

Figure 4. The flowchart of DANN

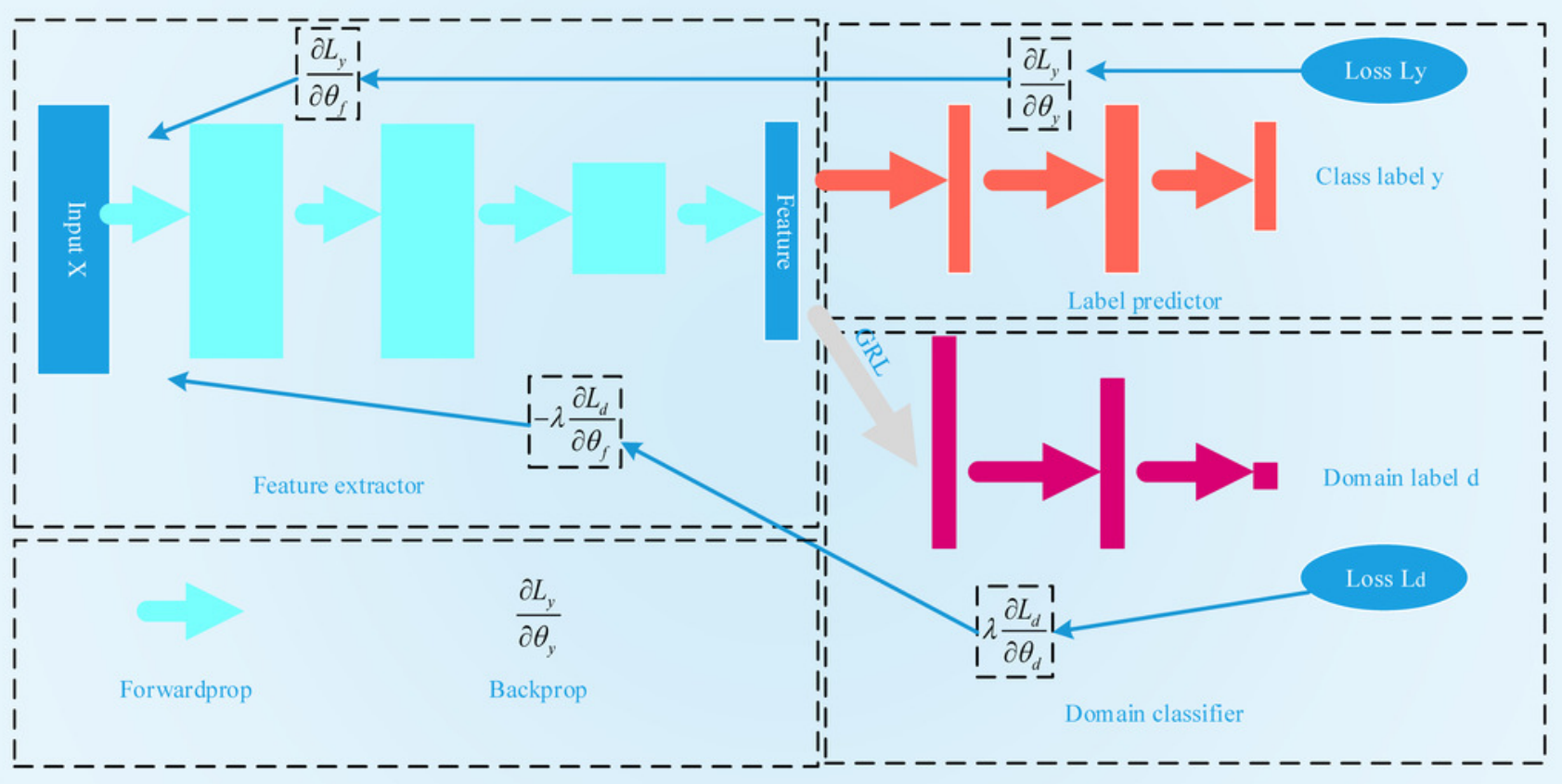


Figure 5

Figure 5. The flowchart of BGRU-DANN

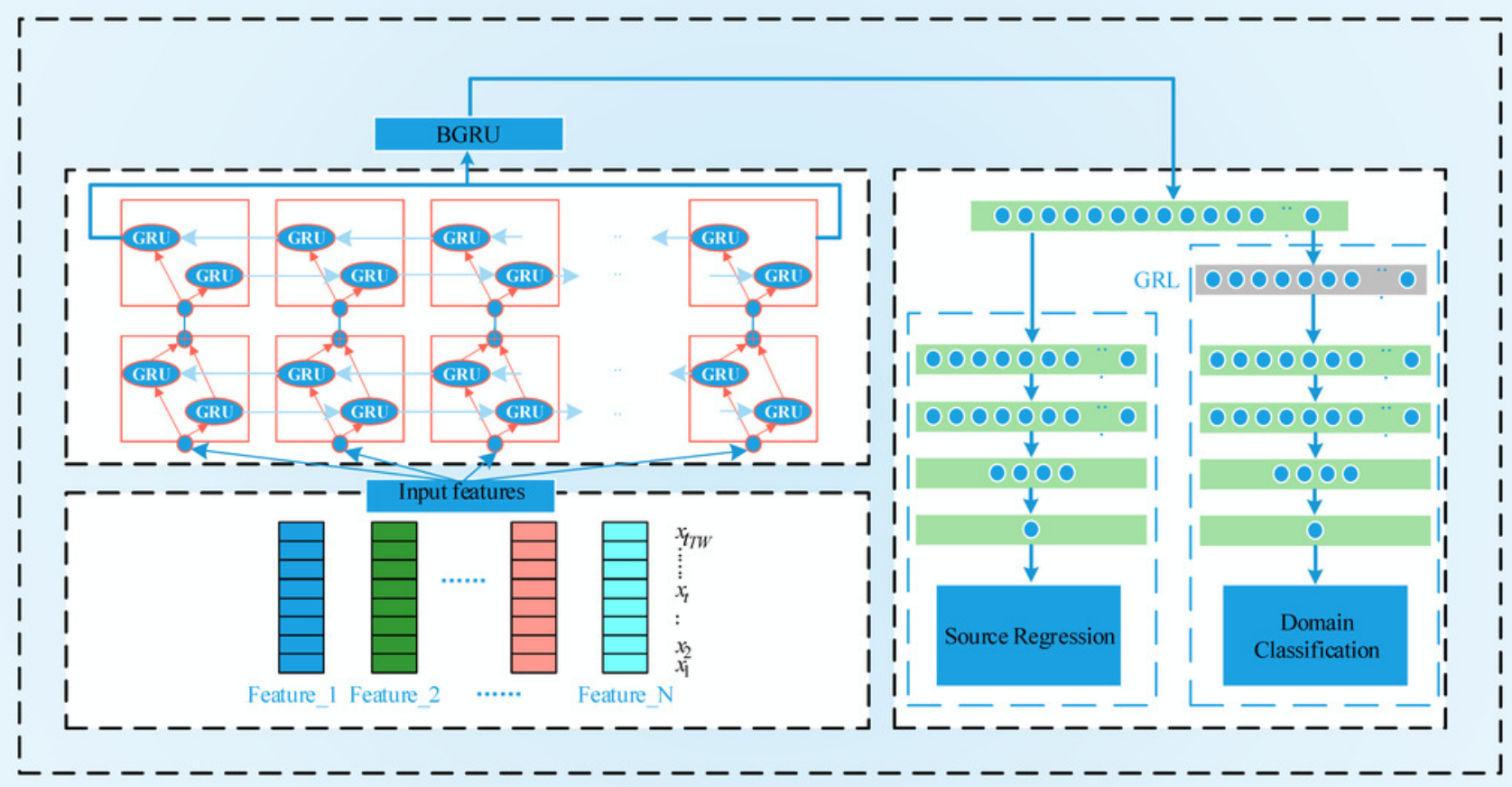


Figure 6

Figure 6. BGRU-DANN Structure

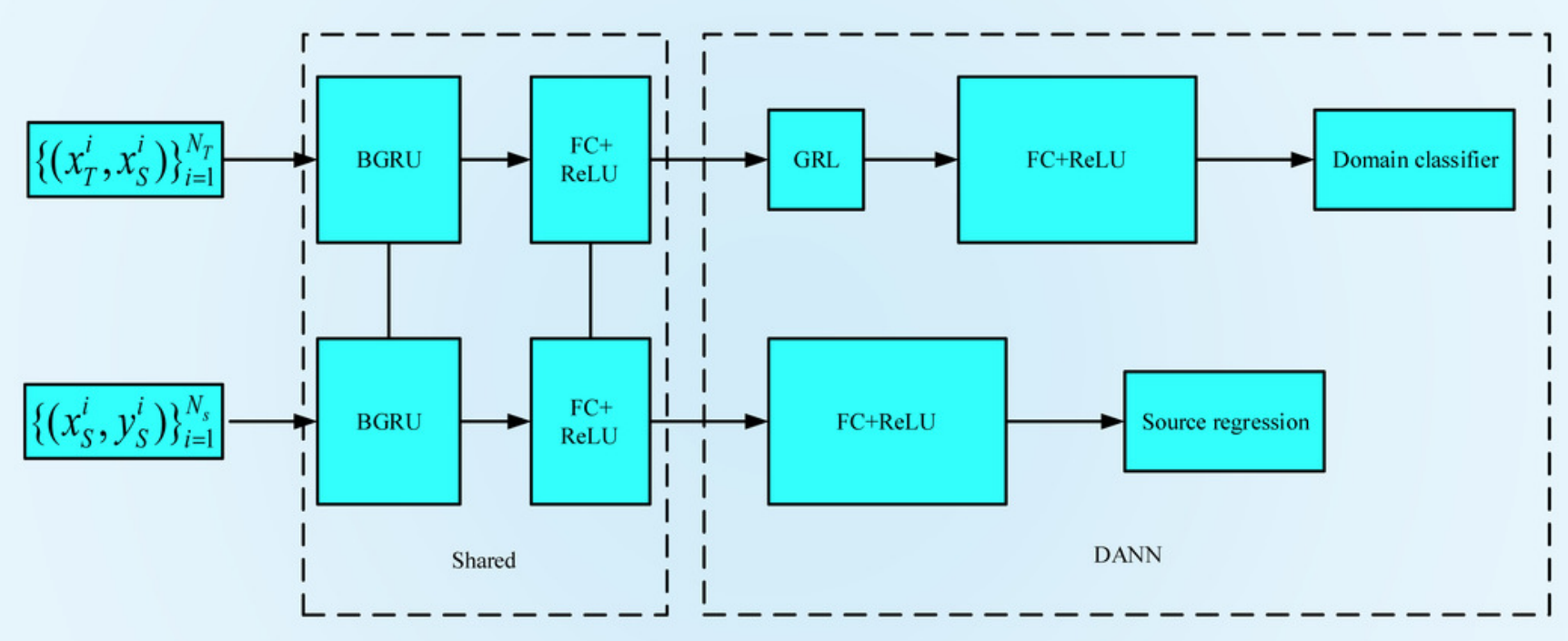




\section{Figure 7}

Figure 7. Prediction results for dataset 2:

(A) Prediction results of Bearing 2-1 using BGRU-DANN; (B) Prediction results of Bearing 2-1 using the comparison method; (C) Prediction results of Bearing 2-4 using BGRU-DANN; (D) Prediction results of Bearing 2-4 using the comparison method; (E) Prediction results of Bearing 2-6 using BGRU-DANN; (F) Prediction results of Bearing 2-6 using the comparison method. 
(A)

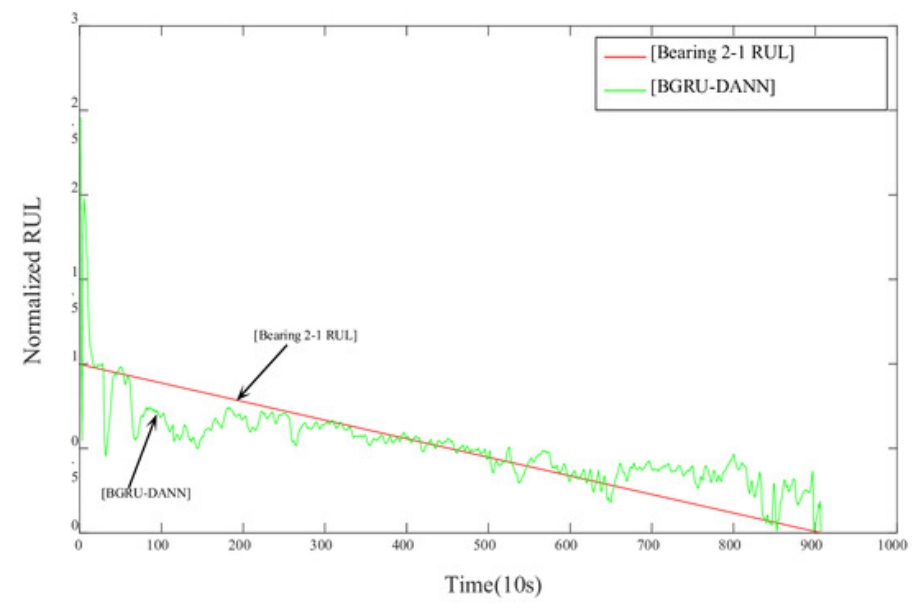

(C)

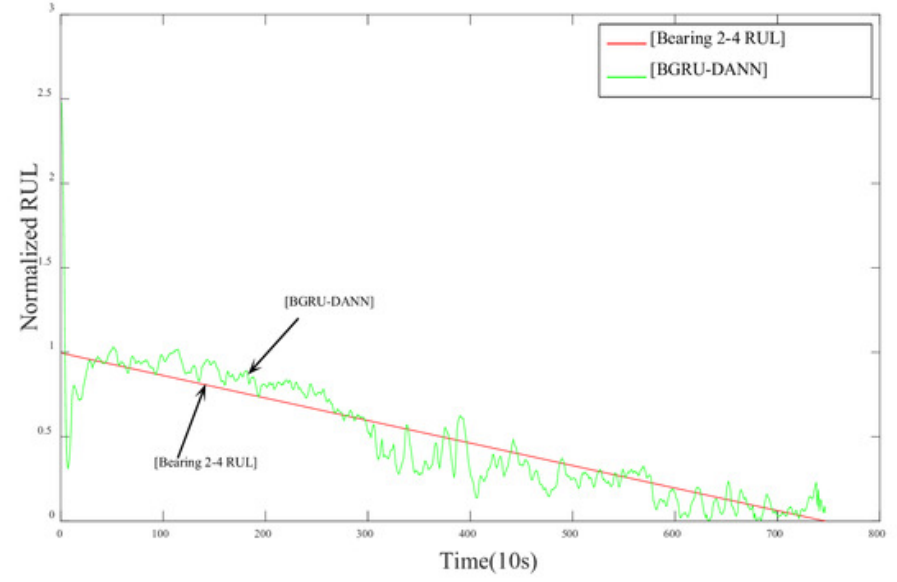

(E)

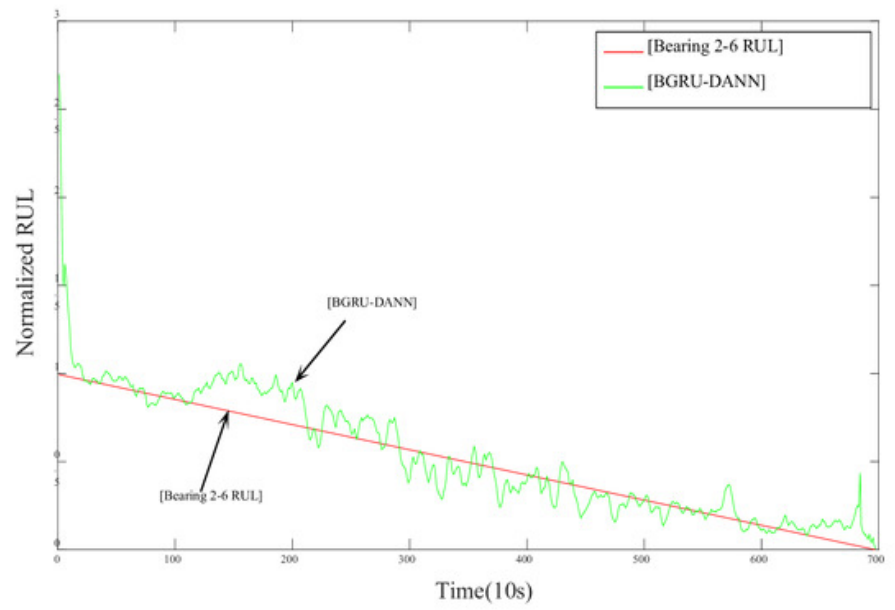

(B)

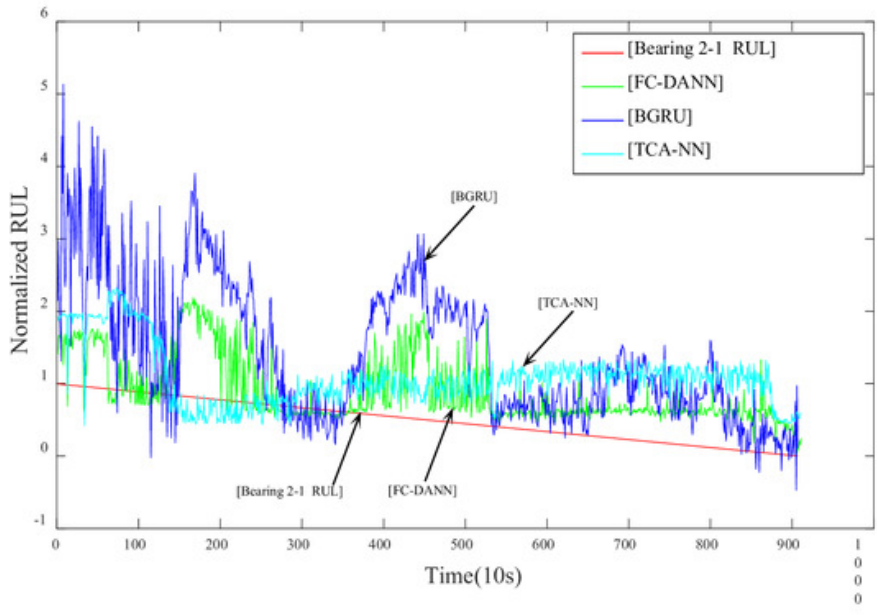

(D)

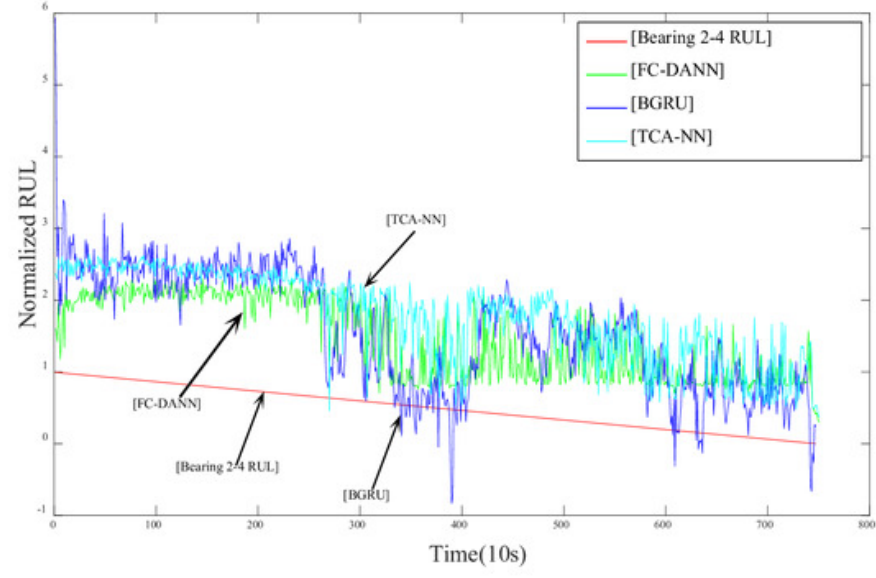

(F)

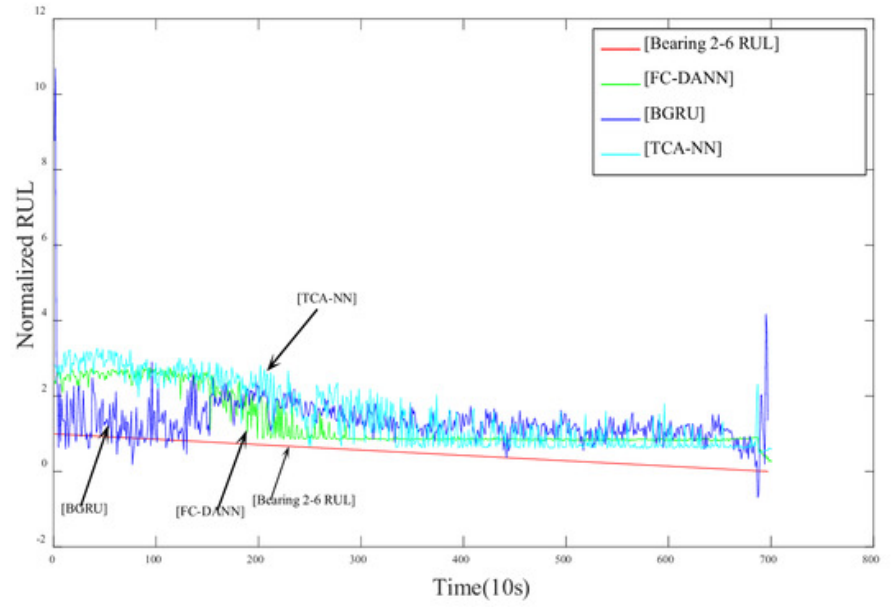


Figure 8

Figure 8. Prediction results for dataset 3:

(A) Prediction results of Bearing 3-1 using BGRU-DANN; (B) Prediction results of Bearing 3-1 using the comparison method; (C) Prediction results of Bearing 3-2 using BGRU-DANN; (D) Prediction results of Bearing 3-2 using the comparison method; (E) Prediction results of Bearing 3-3 using BGRU-DANN; (F) Prediction results of Bearing 3-3 using the comparison method. 
(A)

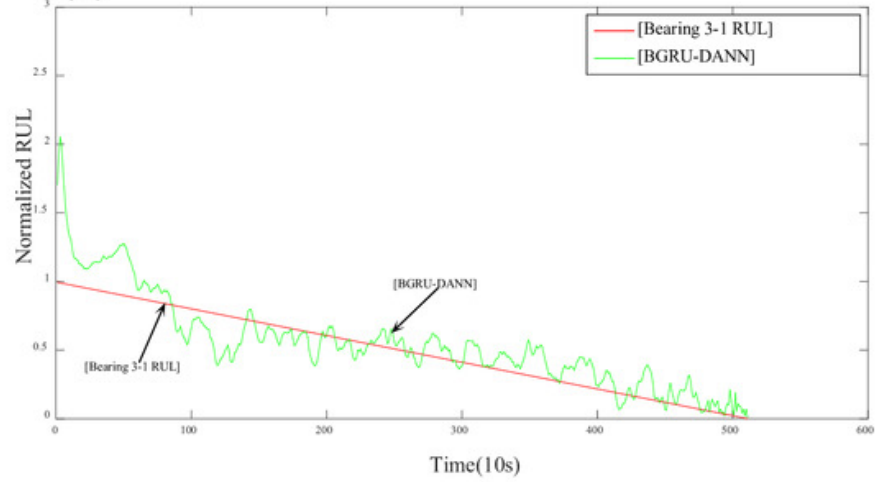

(B)

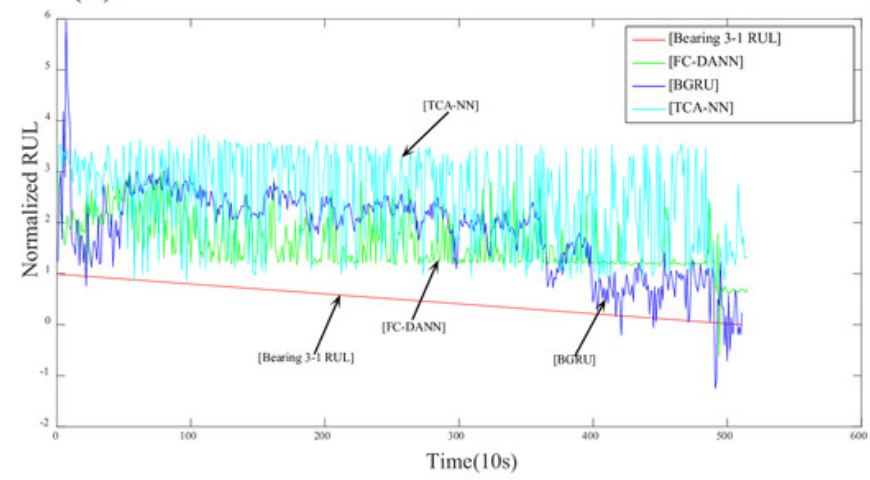

(C)

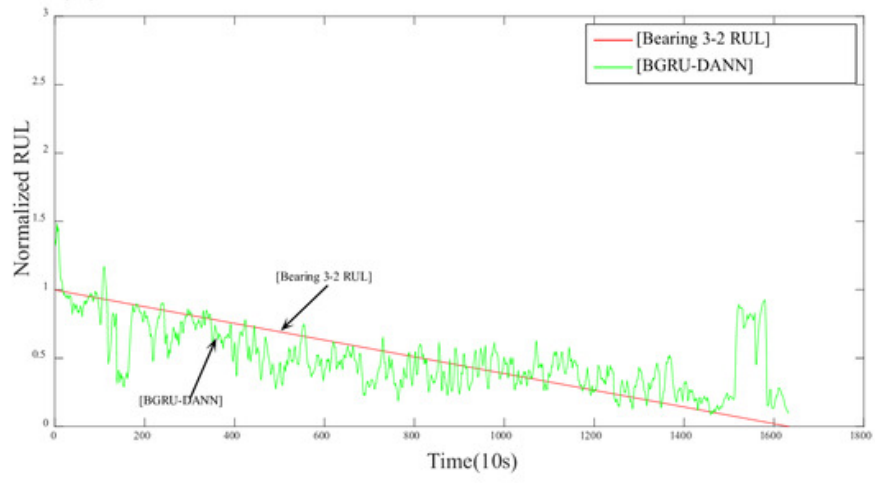

(E)

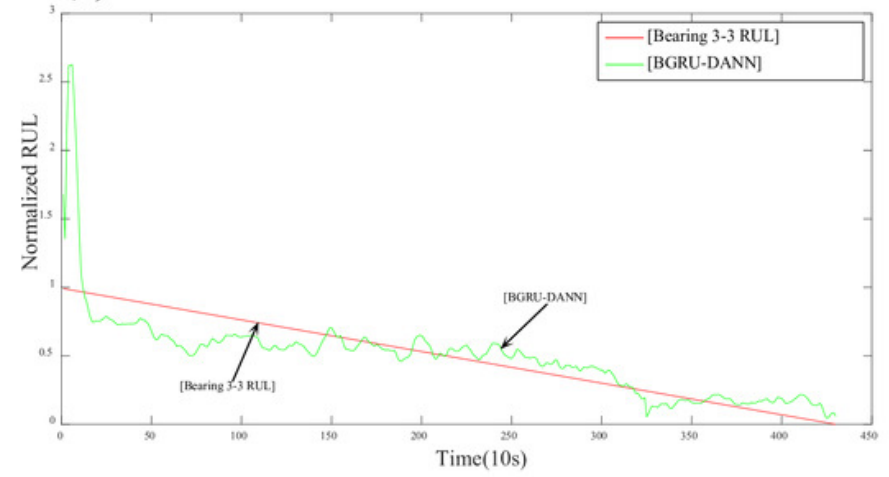

(D)

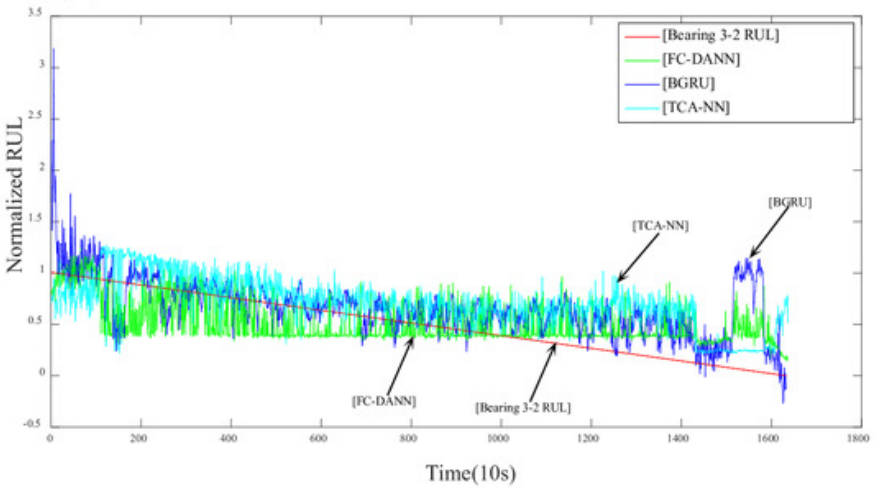

(F)

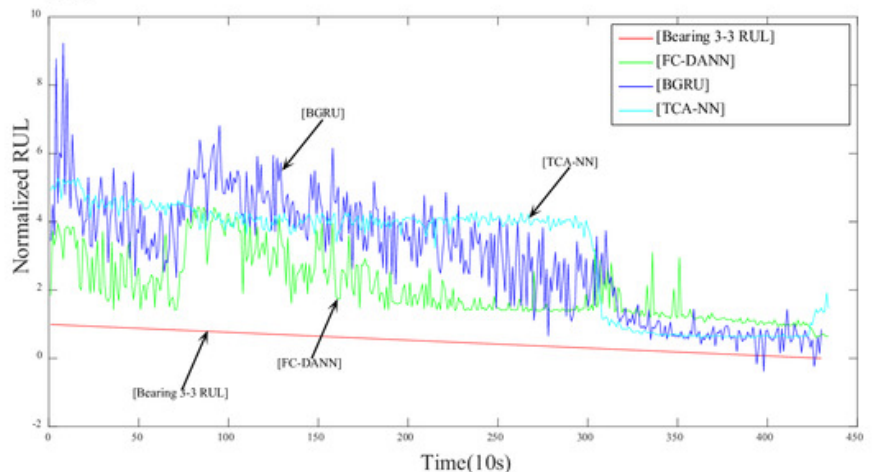


Figure 9

Figure.9 RUL error
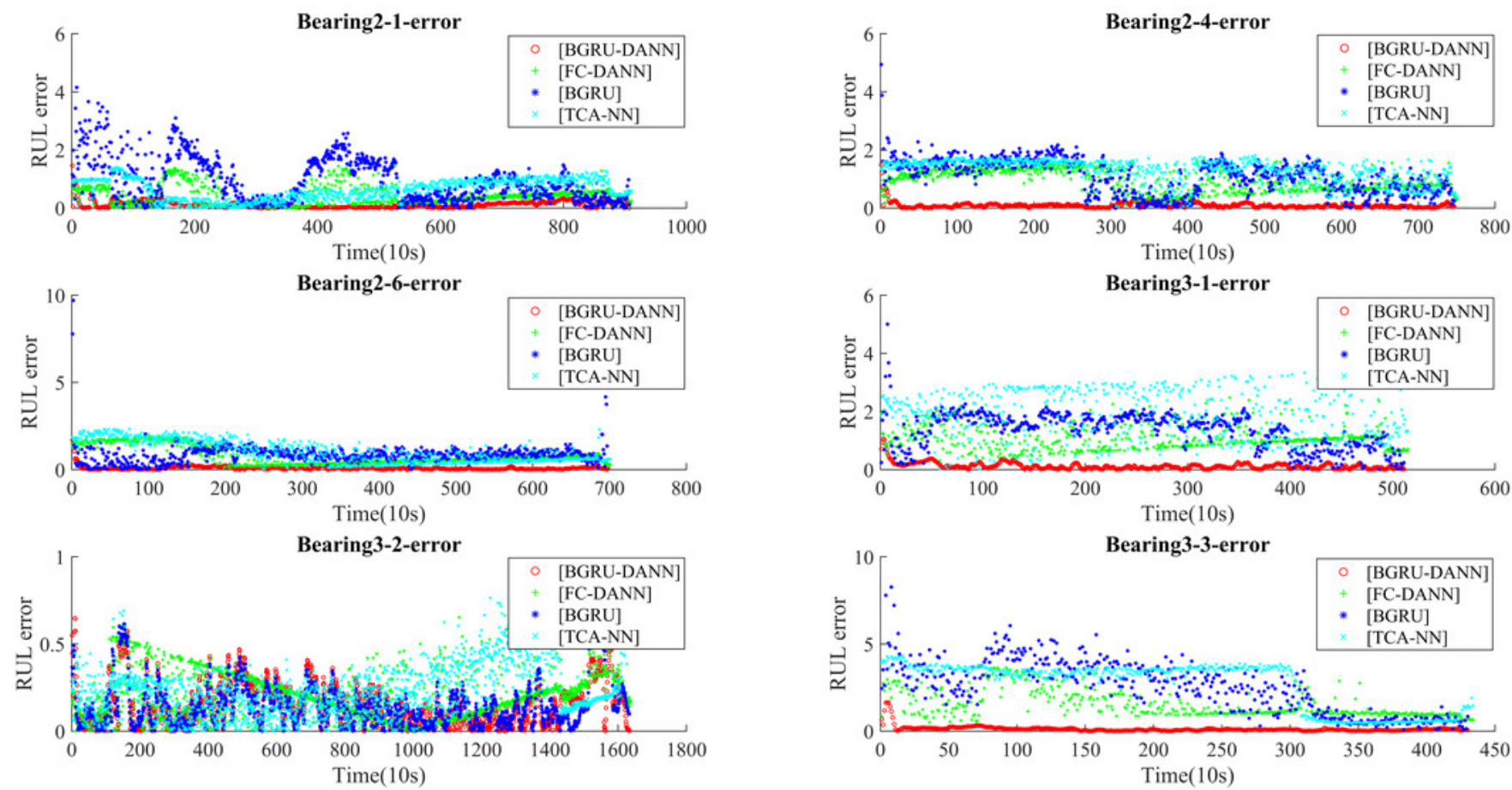